Research Paper

\title{
Surgical Management of the Primary Tumor in Stage IV Colorectal Cancer: A Confirmatory Retrospective Cohort Study
}

\author{
Shahid Ahmed ${ }^{1,2,3}{ }^{凶}$, Anne Leis 3 , Selliah Chandra-Kanthan4, Anthony Fields 5 , Bruce Reeder ${ }^{3}$, Nayyer \\ Iqbal $^{1,2}$, Kamal Haider ${ }^{1,2}$, Duc Le ${ }^{1,2}$, Punam Pahwa ${ }^{3,6}$ \\ 1. Saskatchewan Cancer Agency; \\ 2. Department of Oncology, University of Saskatchewan; \\ 3. Department of Community Health and Epidemiology, University of Saskatchewan; \\ 4. Department of Surgery, University of Saskatchewan; \\ 5. Department of Oncology, University of Alberta; \\ 6. Division of Biostatistics, Department of Community Health and Epidemiology, University of Saskatchewan. \\ $\triangle$ Corresponding author: Shahid Ahmed, MD, FRCPC, FACP. Clinical Professor of Medicine, Site Leader of Gastrointestinal Cancer Group, Saskatoon Cancer Center, 20 \\ Campus Drive, University of Saskatchewan, Saskatoon, SK, Canada S7N4H4 Phone: (306) 655-2710 Fax: (306) 655-0633 E-mail: shahid.ahmed@saskcancer.ca \\ (C) Ivyspring International Publisher. Reproduction is permitted for personal, noncommercial use, provided that the article is in whole, unmodified, and properly cited. See \\ http://ivyspring.com/terms for terms and conditions.
}

Received: 2015.12.16; Accepted: 2016.03.16; Published: 2016.04.27

\begin{abstract}
Background: Observational studies have suggested that patients with stage IV colorectal cancer who undergo surgical resection of the primary tumor (SRPT) have better survival. Yet the results are not confirmed in the setting of a randomized controlled trial. Lack of randomization and failure to control prognostic variables such as performance status are major critiques to the findings of the observational studies. We previously have shown that SRPT, independent of chemotherapy and performance status, improves survival of stage IV CRC patients. The current study aims to validate our findings in patients with stage IV CRC who were diagnosed during the period of modern chemotherapy. Methods: A cohort of 569 patients with stage IV CRC diagnosed during 2006-2010 in the province of Saskatchewan was evaluated. Cox regression model was used for the adjustment of prognostic variables. Results: Median age was 69 years (59-95) and M: F was 1.4:1. Fifty-seven percent received chemotherapy, $91.4 \%$ received FOLFIRI or FOLFOX \& $67 \%$ received a biologic agent. Median overall survival (OS) of patients who underwent SRPT and received chemotherapy was 27 months compared with 14 months of the non-resection group $(\mathrm{p}<0.0001)$. Median OS of patients who received all active agents and had SRPT was 39 months (95\%Cl: $25.1-52.9)$. On multivariate analysis, SRPT, hazard ratio (HR):0.44 (95\%Cl: $0.35-0.56)$, use of chemotherapy, HR: 0.33 (95\% Cl: $0.26-0.43)$, metastasectomy, HR: 0.43 (95\% Cl: $0.31-0.58)$, second line therapy, HR: $0.50(95 \% \mathrm{Cl}: 0.35-0.70)$, and third line therapy, HR: $0.58(95 \% \mathrm{Cl}$ : 0.41-0.83) were correlated with superior survival. Conclusions: This study confirms our findings and supports a favorable association between SRPT and survival in patients with stage IV CRC who are treated with modern therapy.
\end{abstract}

Key words: stage IV colorectal cancer, primary tumor, surgical resection, surgery, survival, chemotherapy, combination chemotherapy, biological agents.

\section{Introduction}

Several observational studies have demonstrated superior survival of patients with stage IV colorectal cancer (CRC) who undergo surgical resection of the primary tumor [SRPT] (1-6). For example, a systematic review and meta-analysis of 15 observational studies involving 12456 patients with stage IV CRC demonstrated a hazard ratio (HR) for mortality of $0.69(95 \%$ confidence interval [CI]: 0.61-0.79) favoring surgery (2). A subsequent meta-analysis involving 44,226 patients in 21 studies 
indicated potential benefit of resection of the primary tumor in patients with unresectable metastases (odd ratio of $0.28 ; 95 \% \mathrm{CI}$ : $0.17-0.47)$, translating into a difference in mean survival of 6.4 months in favor of resection (3). Yet the results are not confirmed in the setting of a randomized controlled trial. Lack of randomization and failure to control prognostic variables that affect survival including systemic therapy and performance status are major critiques to the findings of the observational studies. Consequently the survival benefit related to SRPT has been attributed to selection bias and selection of younger and healthier patients with good performance status.

Our group has demonstrated that surgical resection of the primary tumor improves survival of a cohort of patients with stage IV CRC, who were diagnosed between 1992-2005, irrespective of age, comorbid illness, performance status, chemotherapy, metastasectomy and other important prognostic variables (1). In this group of patients SRPT was associated with $46 \%$ reduction in mortality (HR 0.54, 95\% CI: 0.41-0.58). However, only $42 \%$ of patients were treated with systemic therapy. Among the treated patients about $45 \%$ received irinotecan or oxaliplatin based (FOLFIRI or FOLFOX) chemotherapy. Moreover, less than 5\% patients received a biological agent. It is not known if similar benefit can be achieved with the use of more effective chemotherapy and biological agents in the management of metastatic CRC. We have undertaken the current study to confirm our findings in a cohort of patients with stage IV CRC who were diagnosed during the period of modern systemic therapy.

\section{Objectives}

The study objectives were to validate prognostic impact of SRPT in patients with stage IV CRC who were treated with modern systemic therapy and to assess interaction of SRPT with other prognostic variables in order to identify subgroups of patients who received greater benefit from surgery.

\section{Methods}

\section{Eligibility Criteria}

The study protocol was approved by the University of Saskatchewan's Ethics Board. Individual records of patients $\geq 18$ years, diagnosed with synchronous stage IV CRC between 2006 and 2010 in the province of Saskatchewan were reviewed. Patients with previous history of CRC, with another active second primary cancer, or who had pathology other than adenocarcinoma were excluded.

\section{Statistical Analysis}

The primary end point of this study was to determine relationship between SRPT and overall survival (OS). Survival was estimated by using the Kaplan-Meier method. Survival distribution of subgroups was compared by the Log Rank test.

Charlson comorbidity index was used in this confirmatory study to defined major comorbid illness (7). A multivariate analysis was performed to determine the prognostic significance of SRPT in patients with stage IV CRC in relation to other clinico-pathological variables. The Cox proportional hazard model was used and the hazard ratio (HR) and its $95 \%$ CI were estimated. Following variables were examined with respect to their prognostic significance:

Interventions: Resection of primary tumor, metastasectomy, use of chemotherapy with or without biologics, second line therapy, third line therapy, and radiation therapy; clinical \& demographic variables: age (<65 vs. $\geq 65)$, gender, major comorbid illness (as per Charlson comorbidity index), secondary cancer, Eastern Cooperative Oncology Group performance status [ECOG PS] $(<2$ vs. $\geq 2$ ), cancer center, and smoking; laboratory values: albumin ( $\geq 36$ vs. $<36 \mathrm{~g} / \mathrm{l}$ ), bilirubin ( $\geq 26$ vs. $<26$ $\mathrm{um} / \mathrm{l})$, alkaline phosphatase $(\geq 120$ vs. $<120 \mathrm{U} / \mathrm{l})$, sodium level ( $\leq 135 \mathrm{mEq} / 1$ vs. $>135 \mathrm{mEq} / \mathrm{l})$, serum creatinine ( $\geq 120$ vs. $<120 \mathrm{um} / \mathrm{l})$, blood urea nitrogen (BUN) ( $\geq 8$ vs. $<8 \mathrm{~mm} / \mathrm{l}$ ), hemoglobin ( $\geq 120$ vs. $<120$ $\mathrm{g} / \mathrm{l})$, white blood cell (WBC) ( $\geq 11$ vs. $\left.<11 \times 10^{9} / 1\right)$, platelet count $\left(\geq 450\right.$ vs. $\left.<450 \quad \times 10^{9} / 1\right)$, and carcinoembryonic antigen (CEA) ( $\geq 6$ vs. $<6 \mathrm{ug} / \mathrm{l}$ ); disease characteristics: site (colon vs. rectal), grade (3 vs. <3), symptomatic disease, extra-hepatic metastases, and stage (stage IVa vs. stage IVb disease). For the Cox proportional hazard model, log-log survival curves were used to assess the proportional hazards assumption.

All variables that were significant on univariate analysis with $P<0.05$, were examined in multivariate models. The likelihood ratio test and $t$ test were used to determine if a variable correlates with survival in the model. Test for interaction was performed for SRPT and the other variables that were correlated with survival. In addition, secondary analyses were performed in subgroups of patients with asymptomatic disease, or patients who did not have metastasectomy. A two-sided P-value of $<0.05$ was considered to be statistically significant. For missing data imputation technique was used. All patients were followed till June 2014 when the data entry was closed. The SPSS version 22 was used for statistical analysis (SPSS Inc. Chicago, IL). 


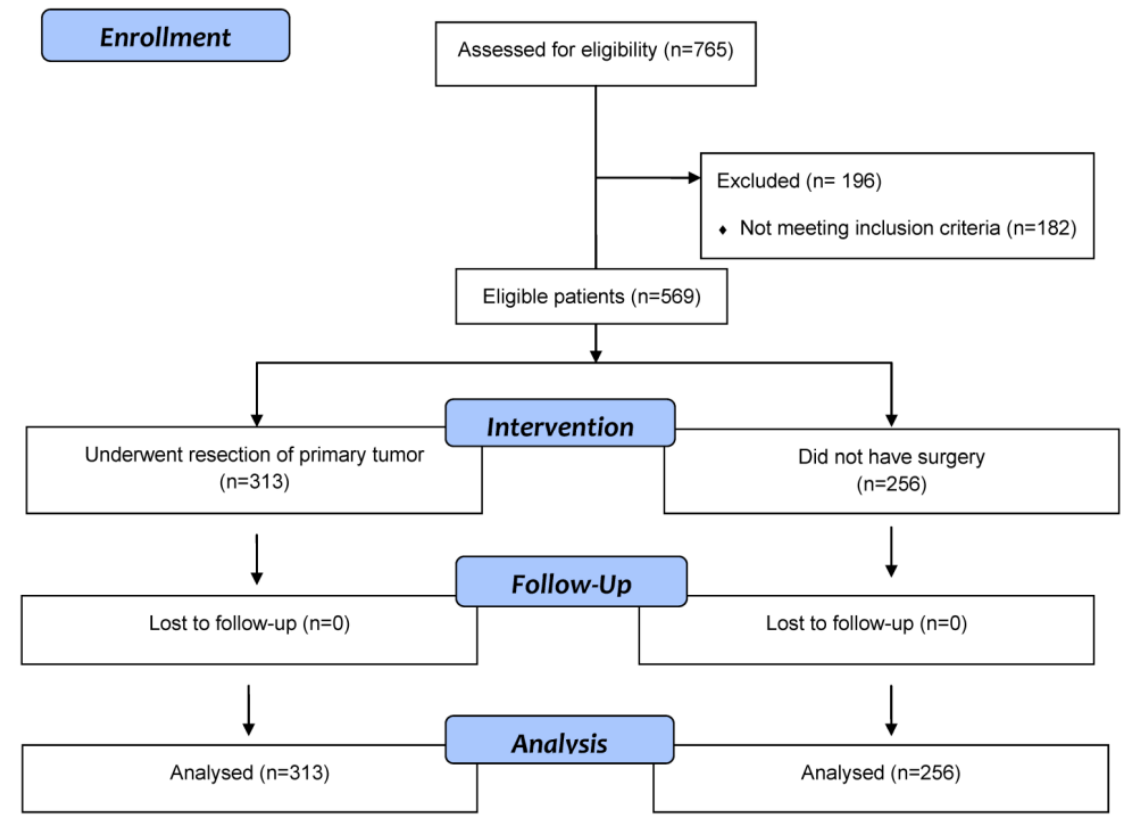

Figure 1: Flow diagram of eligible patients with stage 4 colorectal cancer patients who underwent surgical resection of primary tumor or did not have surgery.

\section{Results}

\section{Baseline Characteristics}

Five hundred and sixty-nine eligible patients with synchronous stage IV CRC were identified (Figure 1). Their median age was 69 years (range: 59-95) and male: female was 1.4:1. Three hundred and thirteen $(55 \%)$ patients underwent surgery and 256 $(45 \%)$ did not have surgery. Of 569 patients, $340(60 \%)$ were $\geq 65$ years and $201(35 \%)$ had ECOG PS of $\geq 2$ (Table 1). Although no significant differences were noted between the two groups with respect to age, gender, comorbid illness, and smoking status, 122 (48\%) patients in the non-resection group had ECOG PS $>1$ compared with $79(25 \%)$ patients in the resection group $(p<0.001)$. In addition, significant differences were noted between the two groups in relationship with serum creatinine, CEA and bilirubin.

\section{Systemic therapy}

Overall $326 \quad(57 \%)$ patients received chemotherapy, $199(64 \%)$ in the resection group and $127(50 \%)$ in the non-resection group $(\mathrm{p}=0.001)$. Of 326 patients, 298 (91\%) received either FOLFIRI (infusional 5 fluorouracil, leucovorin and irinotecan) or FOLFOX (infusional 5 fluorouracil, leucovorin and oxaliplatin), $157(48 \%)$ received both FOLFIRI and FOLFOX, $213(65 \%)$ received bevacizumab, and 34 $(10 \%)$ patients with KRAS wild tumor (about $60 \%$ had KRAS wild disease) received cetuximab or panitumumab. Of 326 patients who were treated with chemotherapy, on progression 174 (53\%) received second line chemotherapy and $52(16 \%)$ received third line therapy. FOLFIRI in combination with bevacizumab was the preferred first line regimen in most patients who were treated with systemic therapy. On progression, FOLFOX was given as second line therapy and panitumumab or cetuximab alone or in combination with irinotecan was used as the standard third line therapy in patients with KRAS wild tumor. There was no significant difference between the two groups in relation to second and third line therapies (Table 1). Furthermore, no significant difference was noted between the two groups with respect to types of systemic therapy, however, $45 \%$ patients in the resection group who were treated with systemic therapy received all available therapy (FOLFIRI, FOLFOX and a biologic agent) compared with $35 \%$ patients in the non-resection group $(p=0.045)$. Of 199 patients in the resection group who received chemotherapy, 172 $(86 \%)$ received chemotherapy after surgery. The median time to start chemotherapy was 79 days (IQR: 64-106).

\section{Disease Characteristics}

Overall about $41 \%$ patients in the non-resection group had rectal or rectosigmoid primary tumor compared with $28 \%$ in the resection group $(p=0.001)$. In addition, approximately $54 \%$ patients in the non-resection group had stage IVb disease compared with $40 \%$ in the resection group ( $p=0.001$ ). Patients in the resection group had significantly more often peritoneal disease compared with patients in the non-resection group who had more often lung or bone metastases. 
Table 1: Characteristics of patients in the entire cohort and subgroups of patients who were treated with surgery and systemic therapy versus systemic therapy alone.

\begin{tabular}{|c|c|c|c|c|}
\hline Variables & $\begin{array}{l}\text { Study } \\
\text { cohort } \\
N=569\end{array}$ & $\begin{array}{l}\text { Resection } \\
\text { group } \\
\mathrm{N}=313(55)\end{array}$ & $\begin{array}{l}\text { Non-resection } \\
\text { group } \mathrm{N}=256 \\
(45)\end{array}$ & $\begin{array}{l}\mathrm{P} \\
\text { value }\end{array}$ \\
\hline \multicolumn{5}{|l|}{ Demographic } \\
\hline Median age & $69(30-95)$ & $68(33-95)$ & $70(30-95)$ & 0.23 \\
\hline Age 65 years or greater & $340(60)$ & $180(58)$ & $160(62)$ & 0.23 \\
\hline $\begin{array}{l}\text { Previous History of } \\
\text { Cancer }\end{array}$ & $82(14)$ & 39 (13) & $43(17)$ & 0.15 \\
\hline $\begin{array}{l}\text { ECOG performance status } \\
2 \text { or more }\end{array}$ & $201(35)$ & $79(25)$ & $122(48)$ & $<0.001$ \\
\hline $\begin{array}{l}\text { Charlson comorbidity } \\
\text { index * }\end{array}$ & $141(25)$ & $85(27)$ & $56(22)$ & 0.056 \\
\hline Male & 335 (59) & $181(58)$ & $154(60)$ & 0.61 \\
\hline Current or ex-smoker & $344(60)$ & $196(63)$ & $148(58)$ & 0.26 \\
\hline \multicolumn{5}{|l|}{ Laboratory values } \\
\hline Albumin $\mathrm{g} / 1$ & $32 \pm 6$ & $33 \pm 7$ & $31 \pm 6$ & 0.39 \\
\hline Alkaline phosphatase U/1 & $202 \pm 277$ & $179 \pm 309$ & $230 \pm 229$ & 0.12 \\
\hline Bilirubin ug/1 & $15 \pm 24$ & $11 \pm 11$ & $18 \pm 34$ & $<0.001$ \\
\hline Blood urea nitrogen $\mathrm{mm} / 1$ & $7 \pm 27$ & $7 \pm 29$ & $8 \pm 24$ & 0.86 \\
\hline CEA ug/1 & $581 \pm 3006$ & $376 \pm 1988$ & $830 \pm 3895$ & 0.004 \\
\hline Creatinine um/1 & $91 \pm 63$ & $86 \pm 31$ & $97 \pm 88$ & $<0.001$ \\
\hline Hemoglobin g/1 & $111 \pm 119$ & $123 \pm 16$ & $118 \pm 17$ & 0.82 \\
\hline Platelet $10^{9} / 1$ & $350 \pm 124$ & $334 \pm 114$ & $368 \pm 135$ & 0.061 \\
\hline $\begin{array}{l}\text { White blood cell count } \\
10^{9} / 1\end{array}$ & $11.3 \pm 47$ & $8.9 \pm 8$ & $10.2 \pm 4$ & 0.61 \\
\hline Sodium meq/1 & $137 \pm 3$ & $138 \pm 3$ & $137 \pm 3$ & 0.27 \\
\hline \multicolumn{5}{|l|}{ Treatment } \\
\hline Chemotherapy & $326(57)$ & $199(64)$ & $127(50)$ & 0.001 \\
\hline Second line therapy & $174(53)$ & $110(55)$ & $64(50)$ & 0.23 \\
\hline Third line therapy & $52(16)$ & $36(18)$ & $16(13)$ & 0.12 \\
\hline FOLFIRI OR FOLFOX & $298(91)$ & $182(92)$ & $116(91)$ & 0.56 \\
\hline Biologics & $218(67)$ & $137(69)$ & $81(64)$ & 0.24 \\
\hline $\begin{array}{l}\text { FOLFIRI \& FOLFOX plus } \\
\text { a biologics }\end{array}$ & $133(41)$ & $89(45)$ & $44(35)$ & 0.045 \\
\hline Radiation therapy & $90(16)$ & $45(14)$ & $45(18)$ & 0.30 \\
\hline Metastasectomy & $89(16)$ & $81(26)$ & $8(3)$ & $<0.001$ \\
\hline \multicolumn{5}{|l|}{ Metastatic sites } \\
\hline Extra-hepatic disease & $305(54)$ & $151(48)$ & $154(60)$ & 0.005 \\
\hline Bone & $21(7)$ & $6(4)$ & $15(10)$ & 0.038 \\
\hline Brain & $5(2)$ & $2(1)$ & $3(2)$ & 0.50 \\
\hline Lung & $125(41)$ & $50(33)$ & $75(49)$ & 0.004 \\
\hline Peritoneum & $124(41)$ & $73(48)$ & $51(33)$ & 0.005 \\
\hline \multicolumn{5}{|l|}{ Tumor } \\
\hline Stage IVb & $264(46)$ & $126(40)$ & $138(54)$ & 0.001 \\
\hline Grade 3 & 151 (27) & $79(25)$ & $72(28)$ & 0.44 \\
\hline Mucinous & 55 (10) & 39 (13) & $16(6)$ & 0.015 \\
\hline $\begin{array}{l}\text { Rectum or rectosigmoid } \\
\text { tumor }\end{array}$ & $190(33)$ & $86(28)$ & $104(41)$ & 0.001 \\
\hline $\begin{array}{l}\text { Symptomatic primary } \\
\text { tumor }\end{array}$ & 224 (39) & $171(55)$ & $53(21)$ & $<0.001$ \\
\hline
\end{tabular}

*Mean Chalrson comorbidity score $9.58 \pm 1.53$ in resection group compared with $9.50 \pm 1.44$ non-resection group. Biologics $=$ cetuximab, panitumumab or bevacizumab; $\mathrm{CEA}=$ carcinoembryonic antigen; $\mathrm{ECOG}=$ Eastern Cooporative Oncology Group; FOLFIRI=Infusional 5-FU, leucovorin and irinotecan; FOLFOX=Infusional 5-FU, leucovorin and oxaliplatin.

\section{Post-operative morbidities and mortality}

Median duration of hospital stay of was 9 days (inter-quartile range: 7-13). Of 313 patients who underwent surgery 74 (24\%) patients developed post-operative complications. Among 74 patients who developed operative complications $65 \%$ had a symptomatic primary tumor. Post-operative complications rates were not mutually exclusive and were as followed: post-operative wound infection in $7 \%$ patients, non-wound infection in 5\% patients, and anastomotic leak, wound dehiscence, bleeding, and pulmonary embolism in $2 \%$ each. Major complications including venous thromboembolism, sepsis, wound dehiscence, anastomotic leakage, post-operative bleeding, pelvic abscess and ischemic bowel were noted in $37(12 \%)$ patients. Of note, 10 (7\%) of 142 patients with asymptomatic or minimally symptomatic tumor developed major complications compared with $27(16 \%)$ of 171 patients with symptomatic primary tumor $(\mathrm{p}=0.01)$.

Overall 30 days mortality rate of the group who underwent surgery was 5\%. Fifteen of 171 (9\%) patients with symptomatic disease compared with 2 $(1 \%)$ of 142 patients with asymptomatic or minimally symptomatic disease died within 30 days of surgery $(\mathrm{p}=0.003)$.

\section{Non-Surgical Interventions}

Overall $30(5 \%)$ patients required a stent for their symptoms. Eight (3\%) of 313 patients who underwent surgery compared with $22(9 \%)$ of 256 patients who did not have surgery required a stent $(p=0.001)$. All 8 patients in the surgery group had the stent placed prior to the surgery. In addition, $38(15 \%)$ patients in the non-resection group developed obstructive symptoms and required a stoma formation (colostomy or ileostomy). Forty-five (14\%) patients in the resection group compared with 45 (17.6) of 256 patients in the non-resection group received radiation therapy $(\mathrm{p}=0.30)$.

\section{Follow up \& Survival}

Median follow up time for the entire cohort was 11 months (inter-quartile range: 2-26 months). No patient was lost to follow up. Median overall survival (OS) of patients who underwent removal of the primary tumor, irrespective of the use of systemic therapy, was 18 months (95\% CI: 15.4-20.6) compared with 4 months (95\% CI: 2.6-5.4) if they did not have surgery $(p<0.001)$. Median overall survival (OS) of patients who underwent SRPT and received chemotherapy was 27 months (95\%CI: 23.3-30.8) versus 14 months (95\%CI: $11.2-16.8)$ if they received chemotherapy but did not have surgery $(p<0.001)$ [Figure 2]. Patients who received second line therapy and underwent SRPT had median OS of 30 months (95\% CI: 24.9-35.1) compared with 20 months (95\% CI: 17.7-22.3) if they did not have surgery $(p<0.001)$ [Figures 3A \& 3B]. About 30\% patients on progression received third line therapy. The median OS of patients who received third line therapy and underwent SRPT was 39 months (95\%CI: 22.1-44.9) compared with 26 months (95\%CI: 10.3-41.7) if they 
did not have surgery $(p=0.13$ ). Median OS of patients who received FOLFIRI and FOLFOX and a biologic agent (bevacizumab or anti-EGFR monoclonal antibodies) and underwent SRPT was 35 months (95\% CI: 30-40) compared with 23 months (95\%CI: 19.8-26.3) if they did not surgery $(p<0.001)$ [Figure 4].
A subgroup of patients with asymptomatic or minimally symptomatic primary tumor who underwent surgery and received systemic therapy had a median OS of 34 months (95\%CI: 26.6-43.4) compared with median OS of 14 months (95\%CI: 11.1-17.0) if they did not have surgery $(p<0.001)$.

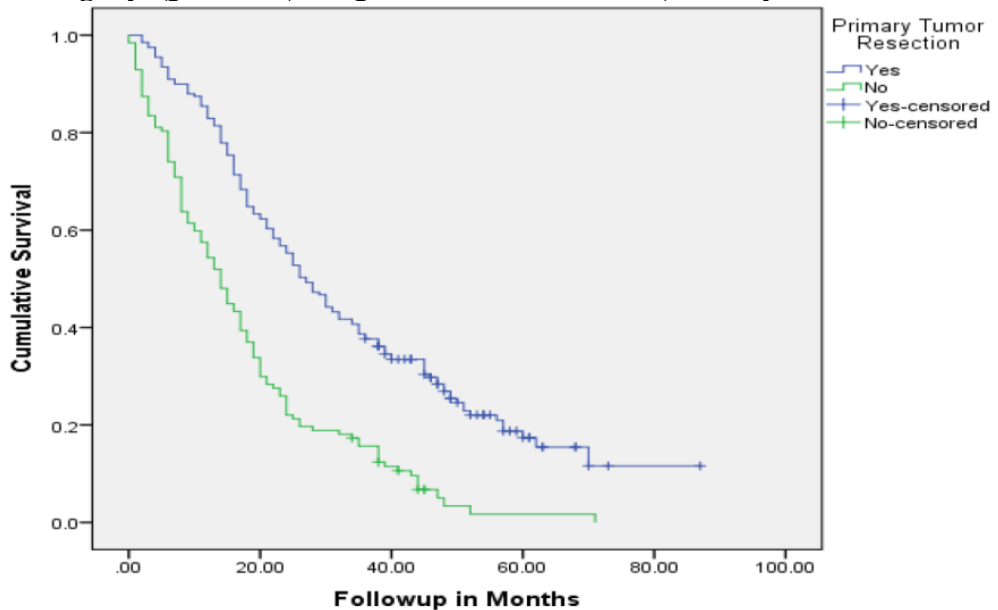

Figure 2: Overall survival of patients who underwent surgery compared with no surgical intervention.
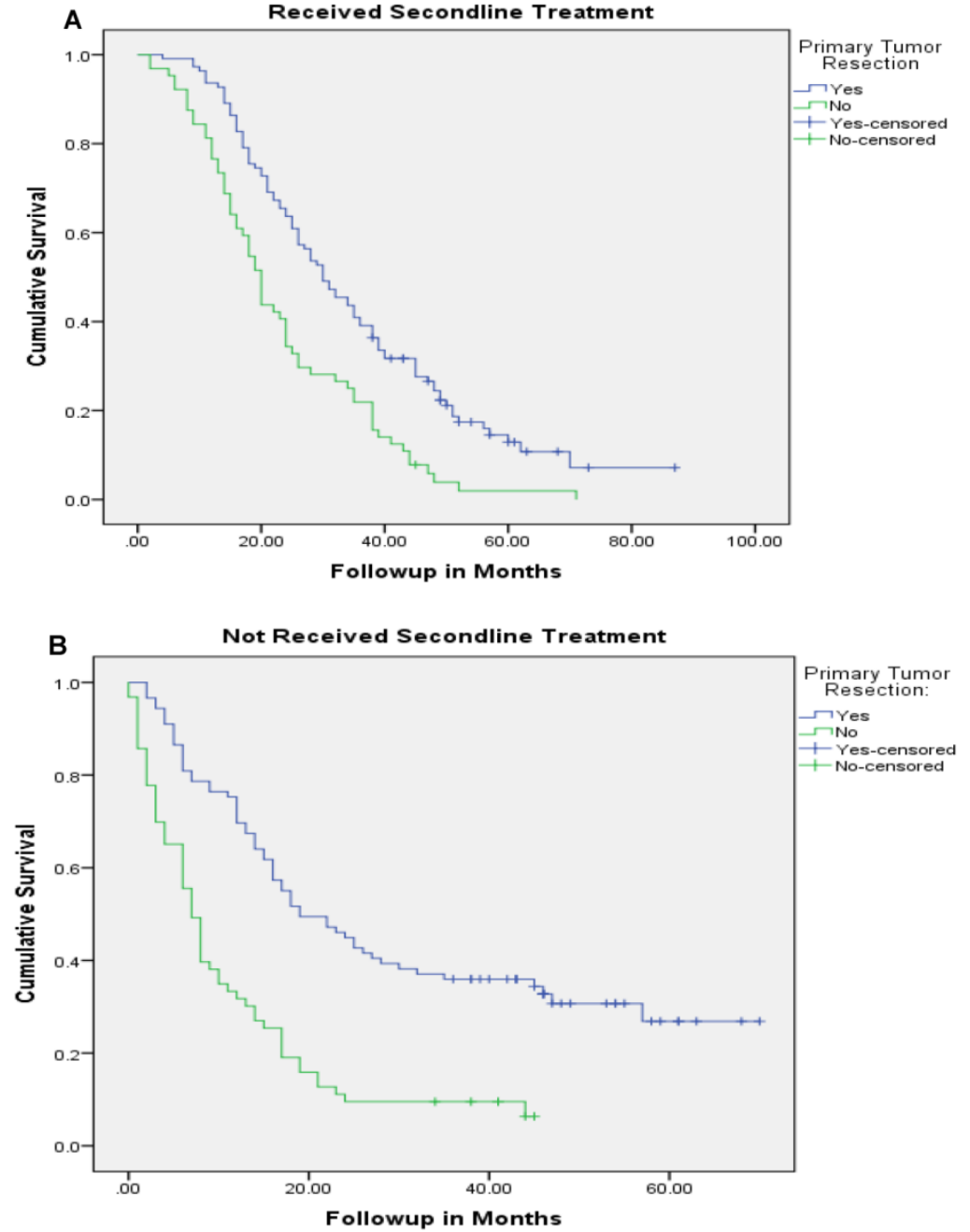

Figure 3: Overall survival of patients who received second line therapy and underwent surgery of the primary tumor (2A) versus if they did not receive second line therapy (3B). 


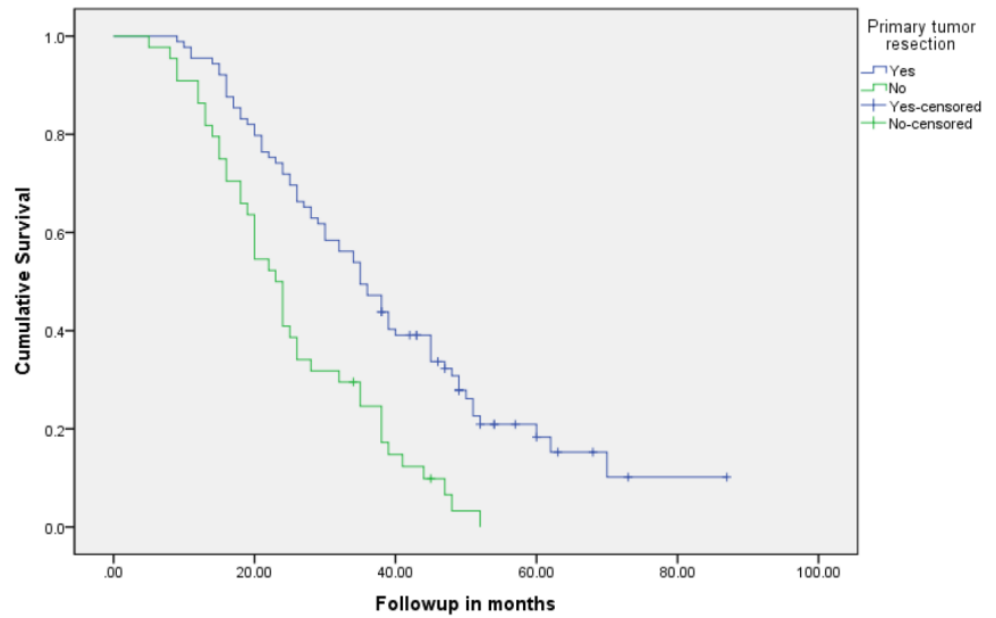

Figure 4: Overall survival of patients with stage IV colorectal cancer who were treated with combination of chemotherapy and biologics and underwent surgery compared with no surgery.

Table 2: Relationship between various clinic-pathological variables and survival in univariate analysis.

\begin{tabular}{|c|c|c|c|c|}
\hline Variables & $\mathrm{HR}(95 \% \mathrm{CI})$ & P value & $\begin{array}{l}\text { HR }(95 \% \text { CI) for } \\
\text { Interaction with } \\
\text { SPRT }\end{array}$ & P value \\
\hline Age $\geq 65$ years & $1.87(1.56-2.25)$ & $<0.001$ & $1.36(0.95-1.94)$ & 0.096 \\
\hline Male gender & $0.90(0.75-1.10)$ & 0.22 & $1.17(0.83-1.67)$ & 0.37 \\
\hline ECOG PS & $2.51(2.09-3.02)$ & $<0.001$ & $0.94(0.65-1.36)$ & 0.75 \\
\hline Comorbid illness & $1.50(1.23-1.85)$ & $<0.001$ & $1.11(0.74-1.67)$ & 0.63 \\
\hline Current Smoking & $0.86(0.69-1.08)$ & 0.20 & $0.80(0.52-1.26)$ & 0.35 \\
\hline Ex-Smoking & $0.91(0.76-1.09)$ & 0.31 & $1(0.70-1.43)$ & 0.98 \\
\hline Treatment Centers & $1.04(0.87-1.23)$ & 0.68 & $1.33(0.94-1.89)$ & 0.11 \\
\hline Albumin $<35 \mathrm{~g} / 1$ & $1.95(1.60-2.39)$ & $<0.001$ & $0.76(0.49-1.19)$ & 0.23 \\
\hline $\begin{array}{l}\text { Alkaline phosphatase } \\
>120 \mathrm{U} / 1\end{array}$ & $2.17(1.79-2.62)$ & $<0.001$ & $1.04(0.70-1.55)$ & 0.85 \\
\hline Bilirubin $\geq 26 \mathrm{um} / 1$ & $1.77(1.28-2.46)$ & 0.001 & $1.93(0.97-3.87$ & 0.06 \\
\hline $\mathrm{BUN} \geq 8 \mathrm{~mm} / 1$ & $1.48(1.13-1.93)$ & 0.007 & $1.82(1.05-3.16)$ & 0.034 \\
\hline $\mathrm{CEA} \geq 5 \mathrm{ug} / 1$ & $2.11(1.63-2.76)$ & $<0.001$ & $1.18(0.64-2.20)$ & 0.60 \\
\hline Creatinine $\geq 120 \mathrm{um} / 1$ & $1.46(1.06-1.99)$ & 0.026 & $1.36(0.72-2.57)$ & 0.34 \\
\hline Hemoglobin $<120 \mathrm{~g} / 1$ & $1.82(1.49-2.22)$ & $<0.001$ & $0.90(0.60-1.34)$ & 0.60 \\
\hline $\begin{array}{l}\text { Platelet count, } \geq 450 \\
\text { x } 10^{9} / 1\end{array}$ & $0.92(0.73-1.17)$ & 0.51 & $1.14(0.70-1.86)$ & 0.60 \\
\hline$W B C \geq 11 \times 10^{9} / 1$ & $1.39(1.12-1.71)$ & 0.004 & $1.30(0.84-2.01)$ & 0.23 \\
\hline Sodium $<135 \mathrm{meq} / 1$ & $1.19(0.95-1.48)$ & 0.14 & $1.02(0.65-1.60)$ & 0.92 \\
\hline Extra-hepatic disease & $1.21(1.02-1.44)$ & 0.031 & $1.36(0.96-1.94)$ & 0.08 \\
\hline Grade 3 & $1.48(1.22-1.79)$ & $<0.001$ & $1.34(0.91-1.97)$ & 0.14 \\
\hline Mucinous pathology & $1.13(0.85-1.51)$ & 0.40 & $1.74(0.92-3.28)$ & 0.09 \\
\hline Rectum & $0.80(0.66-0.96)$ & 0.014 & $0.84(0.58-1.24)$ & 0.39 \\
\hline Stage IVb disease & $1.38(1.16-1.64)$ & $<0.001$ & $1.43(1.01-2.03)$ & 0.04 \\
\hline $\begin{array}{l}\text { Symptomatic primary } \\
\text { tumor }\end{array}$ & $1.08(0.90-1.28)$ & 0.42 & $1.05(0.71-1.55)$ & 0.82 \\
\hline $\begin{array}{l}\text { Primary tumor } \\
\text { resection }\end{array}$ & $0.44(0.37-0.53)$ & $<0.001$ & NA & NA \\
\hline Metastasectomy & $0.30(0.22-0.40)$ & $<0.001$ & $0.74(0.58-0.94)$ & 0.015 \\
\hline Chemotherapy & $0.24(0.20-0.29)$ & $<0.001$ & $1.36(0.95-1.94)$ & 0.09 \\
\hline Second line Treatment & 0.44 & 0.025 & $2.10(1.28-3.35)$ & 0.003 \\
\hline Third line Treatment & $0.41(0.30-0.56)$ & $<0.001$ & $1.77(0.89-3.49)$ & 0.10 \\
\hline FOLFOX or FOLFIRI & $0.27(0.23-0.33)$ & $<0.001$ & $1.49(1.04-2.12)$ & 0.029 \\
\hline Biologics & $0.34(0.28-0.41)$ & $<0.001$ & $1.44(1.0-2.08)$ & 0.05 \\
\hline Radiation & $0.66(0.52-0.84)$ & $<0.001$ & $0.97(0.60-1.57)$ & 0.90 \\
\hline
\end{tabular}

\section{Cox Proportional multivariate modeling for survival}

One univariate analyses several variables were correlated with survival (Table 2). Tests for interaction were significant between resection of primary tumor and metastasectomy, second line therapy, stage IVb disease and elevated BUN. On multivariate analysis SRPT independently correlated with superior survival with HR for mortality of 0.44 (95\%CI: 0.35-0.56). In addition, chemotherapy, HR 0.33 (95\%CI: 0.26-0.43), metastasectomy, HR 0.43 (95\%CI: 0.31-0.58), second line therapy, HR 0.50 (95\% CI:0.35-0.70), and third line therapy, HR 0.58 (95\%CI:0.41-0.83) were correlated with superior survival whereas elevated alkaline phosphatase, HR 1.50 (95\%CI:1.20-1.78), grade 3 tumor, HR 1.33 (95\%CI:1.10-1.62), leukocytosis, HR 1.32 (95\%CI:1.05-1.66), stage IVb disease, HR 1.31 (95\%CI:1.10-1.56), and ECOG PS >1, HR 1.30 (95\% CI:1.04-1.57) were correlated with inferior survival (Table 3).

After adjustment for other prognostic variables only the interaction between SRPT and subsequent line of therapy was significant suggesting a differential benefit of removal of primary tumor in patients who received other line of therapies. Patients who underwent SRPT and received second line therapy had a median OS of 30 months (95\% CI:24.9-35.1) compared with 20 months (95\%CI:17.7-22.3) if they did not have surgery ( $p$ $<0.001)$. Conversely, patients who had SRPT but did not receive second line therapy had a median OS of 19 months (95\% CI: 11.8-26.2) compared with 7 months (95\% CI: 5.4-8.6) if they did not have surgery $(\mathrm{P}<0.001)$. 


\section{Secondary analyses}

In the secondary multivariate analyses that excluded the patients who underwent metastasectmy, SRPT was independently associated with significant reduction in mortality with $\mathrm{HR}, 0.48$ (95\% CI: $0.38-0.61$ ). Likewise, in a subgroup of 345 patients with asymptomatic or relatively asymptomatic primary tumors, SRPT was significantly correlated with better survival with HR 0.32 (95\%CI: 0.22-0.45). Similar benefits were noted in sub-cohorts of patients with colon cancer or ECOG performance status of 0 or 1. In the subgroup of 379 patients with colon cancer surgery independently correlated with survival with HR for mortality of 0.39 (95\% CI: 0.29-0.53). After excluding patients with poor performance status resection of the primary tumor remained an independent prognostic variable with $\mathrm{HR}$ of 0.48 (95\% CI: 0.35-0.66).

Table 3: Relationship between various clinic-pathological variables and survival in multivariate analysis.

\begin{tabular}{ll}
\hline Variables & HR $(95 \% \mathrm{CI})$ \\
\hline Surgical resection of primary tumor & $0.44(0.35-0.56)$ \\
Yes & 1 \\
No & $0.33(0.26-0.43)$ \\
Chemotherapy & 1 \\
Yes & \\
No & $0.50(0.35-0.70)$ \\
Second line therapy & 1 \\
Yes & \\
No & $0.58(0.41-0.83)$ \\
Third line therapy & 1 \\
Yes & \\
No & $1.50(1.20-1.78)$ \\
Alkaline phosphatase $>120 \mathrm{U} / 1$ & 1 \\
Yes & \\
No & $1.33(1.10-1.62)$ \\
Grade 3 tumors & 1 \\
Yes & \\
No & $1.32(1.05-1.66)$ \\
Leukocytosis & 1 \\
Yes & \\
No & $1.31(1.10-1.56)$ \\
Stage IVb disease & \\
Yes & \\
No & $1.30(1.04-1.57)$ \\
ECOG performance status $>1$ & 1 \\
Yes &
\end{tabular}

\section{Discussion}

The present study confirmed the survival benefit of SRPT in patients who were treated with combination chemotherapy and biologics. In addition to surgery of the primary tumor, systemic therapy, subsequent line of therapies, metastasectomy, elevated alkaline phosphatase, grade 3 tumor, leukocytosis, stage IVb disease, and performance status were correlated with survival. A significantly higher proportion of patients in the non-resection group had low performance status or larger number of metastatic sites involvement. Furthermore, there was imbalance between the two groups in relation to combination therapy and biologics. Nonetheless, when these variables were included in a multivariate model, SRPT remained an important independent prognostic factor. In fact, the survival benefit was stronger than the other well-established prognostic factors in stage IV CRC including performance status, alkaline phosphatase levels, and the number of metastatic sites $(8,9)$.

The median overall survival of patients with stage IV cancer, who are treated with combination of chemotherapy and biologics in the recent phase 3 clinical trials, has been reported in the range of 24-30 months (10-12). However, our cohort was comprised of real world patients and was inclusive of patients with poor performance status and major comorbid illness. Although the median overall survival of the entire cohort was 11 months (8.8-13.2), the survival of patients who underwent SRPT and received chemotherapy was 27 months (95\%CI: 23.3-30.8). Moreover, patients who underwent SRPT and received second and third line therapy had median OS of 30 months (95\%CI: 24.9-35.1) and 39 months (95\% CI: 22.1-44.9), respectively.

Recent literature also supports survival benefit of SRPT in the era of modern chemotherapy. A retrospective analysis of CAIRO study that compared combination versus sequential chemotherapy demonstrated a significantly better median OS of 16.7 months in patients who underwent SRPT compared with 11.4 months with no surgery (HR 0.61, 95\%CI:49-0.76) [6]. Likewise, a pool analysis of four French phase 3 trials involving 850 patients indicated survival benefit of surgery (4). More than two third of patients were treated with FOLFIRI or FOLFOX and about $12 \%$ received bevacizumab. The primary tumor resection was an independent predictor of superior survival (HR: $0.63,95 \% \mathrm{CI}$ : 0.53-0.75). In addition our group' meta-analysis of fifteen observational studies did not reveal a positive interaction between surgery and type of chemotherapy.

In agreement with previous observation, patients with asymptomatic or minimally symptomatic disease following surgery had longer survival compared with patients with symptomatic primary tumor (13). Of note patients with symptomatic primary tumor had 30 days post-operative mortality rates of $8.8 \%$ compared $1.5 \%$ if they had an elective surgery. Emergency surgery has consistently been demonstrated to be an important risk factor for inferior outcome in colorectal 
surgery (14). We believe that in addition to the biology of symptomatic disease, the 30 days mortality rates most likely accounted for the differences in survival between the two groups. In consistent with our previous observation, significantly higher number of patients with colon tumor underwent surgery compared with patients with rectal cancer. However, compared with 12992-2005 cohort, patients with rectal cancer did not have better prognosis.

Among the prognostic factors, systemic therapy was the most important prognostic variables. It is known that survival of patients with stage IV CRC is better if they are exposed to all available active therapeutic agents during the course of their disease (15). The subgroup of patients who received combination chemotherapy with bevacizumab and underwent SRPT had median survival of 35 months (95\% CI: 30-40) whereas patients with KRAS wild tumor who received FOLFIRI \& FOLFOX in combination with bevacizumab and subsequently were treated with an anti-EGFR monoclonal antibody had a median OS of 39 months (95\%CI: 25.1-52.9).

Due to presence of multicollinearity between various lines of chemotherapy and the type of regimens, these variables were not fitted together in the final model. A secondary analysis using chemotherapy regimens and biological agents as opposed to the subsequent lines of therapies was performed (not reported). Both combination of chemotherapy (HR: 0.67, 95\%CI: 0.52-0.87) and biological agents (HR:0.60, 95\%CI:0.45-0.78) were independently correlated with superior survival. Of significant importance, when various chemotherapy regimens were fitted in the multivariate model, resection of primary tumor was independently correlated with survival.

Our study revealed positive interaction between SRPT and second line therapy. The patients who had surgery and received second line therapy had a median OS of 30 months compared with 19 months if they did not receive second line therapy $(p=0.005)$. With access to novel agents and their efficacy in the primary tumor as well as lack of major complications related to an intact primary tumor, SRPT is less commonly performed (16-18). Our results, however, support potential benefit of surgery in patients who are treated with modern chemotherapy and suggest a greater benefit in patients who are treated with subsequent line of therapy. Our study is unique that it included performance status, an important prognostic variables in metastatic $C R C$, in the multivariate model. Most population based studies that support benefit of surgery lack individual patients' data and have failed to include performance status in the multivariate models.
In summary the current study suggests survival benefit of SRPT in patients with advanced CRC. To date, no randomized trial has reported survival impact of SRPT in stage IV CRC. Only a prospective randomized trial could confirm the survival benefit conferred by the primary tumor resection. Such trials are ongoing in Europe and will be important to solve this very important question in the management of stage IV CRC.

\section{Acknowledgement}

The study was supported by a research grant from Saskatchewan Cancer Agency. We would like to thanks to Dr. Bonnie Janzen, Mrs. Carla Woites, Dr. Tong Zhu, and Saskatchewan Cancer Agency for their support for this project.

Part of the data was presented at 2015 Gastrointestinal Cancers Symposium, San Francisco, CA, USA.

\section{Competing Interests}

The authors have declared that no competing interest exists.

\section{References}

1. Ahmed S, Leis A, Fields A, et al. Survival impact of surgical resection of primary tumor in patients with stage IV colorectal cancer: Results from a large population-based cohort study. Cancer. 2014;120:683-91.

2. Ahmed S, Shahid RK, Leis A, et al. Should non-curative resection of primary tumor be performed in patients with stage IV colorectal cancer? A systematic review \& meta-analysis. Current Oncology 2013;20:e420-41.

3. Clancy C, Burke JP, Barry M, et al. A Meta-Analysis to Determine the Effect of Primary Tumor Resection for Stage IV Colorectal Cancer with Unresectable Metastases on Patient Survival. Ann Surg Oncol. 2014;21:3900-8.

4. Faron M, Pignon J, Malka D, et al. Is primary tumor resection associated with survival improvement in patients with colorectal cancer and unresectable synchronous metastases? A pooled analysis of individual data from four randomised trials. Eur J Cancer. 2015;51:166-76.

5. Tsang WY, Ziogas A, Lin BS, et al. Role of primary tumor resection among chemotherapy-treated patients with synchronous stage IV colorectal cancer: a survival analysis. J Gastrointest Surg. 2014;18:592-8.

6. Venderbosch S, Wilt JH, Teerenstra S, et al. Prognostic Value of Resection of Primary Tumor in Patients with Stage IV Colorectal Cancer: Retrospective Analysis of Two Randomized Studies and a Review of the Literature. Ann Surg Oncol. 2011;18:3252-60.

7. Hall WH. Ramachandran R, Narayan S, et el. An electronic application for rapidly calculating Charlson comorbidity socre. BMC Cancer. 2004;4:94.

8. Assersohn, L., Norman, A., Cunningham, D. et al. Influence of metastatic site as an additional predictor for response and outcome in advanced colorectal carcinoma. Br J Cancer. 1999;79:1800-05.

9. Köhne, C.H., Cunningham, D., Di, C.F. et al. Clinical determinants of survival in patients with 5-fluorouracil-based treatment for metastatic colorectal cancer: results of a multivariate analysis of 3825 patients. Ann Oncol. 2002;13:308-17.

10. Ahmed S, Johnson $\mathrm{K}$, Ahmed O, Iqbal N. Advances in the management of colorectal cancer: from biology to treatment. Int J Colorectal Dis. 2014;29:1031-42.

11. Venook AP, Niedzwiecki D, Lenz H, et al. CALGB/SWOG 80405: Phase III trial of irinotecan/5-FU/leucovorin (FOLFIRI) or oxaliplatin/5-FU/ leucovorin (mFOLFOX6) with bevacizumab (BV) or cetuximab (CET) for patients (pts) with KRAS wild-type (wt) untreated metastatic adenocarcinoma of the colon or rectum (MCRC). J Clin Oncol 2014;32(suppl): abstLBA3

12. Loupakis F, Cremolini C, Masi G, et al. Initial Therapy with FOLFOXIRI and Bevacizumab for Metastatic Colorectal Cancer. N Engl J Med 2014;371:1609-18

13. Ahmed S, Fields A, Pahwa P, et al. Surgical Resection of Primary Tumor in Asymptomatic or Minimally Symptomatic Patients With Stage IV Colorectal Cancer: A Canadian Province Experience. Clin Colorectal Cancer. 2015;14:e41-7.

14. Gooiker GA, Dekker JW, Bastiaannet E, et al. Risk Factors for Excess Mortality in the First Year After Curative Surgery for Colorectal Cancer. Ann Surg Oncol. 2012;19: 2428-34. 
15. Grothey A, Sargent D, Goldberg RM, et al. Survival of patients with advanced colorectal cancer improves with the availability of fluorouracil-leucovorin, irinotecan, and oxaliplatin in the course of treatment. J Clin Oncol. 2004;22:1209-14.

16. Schrag D, Weiser MR, Goodman KA,Neoadjuvant chemotherapy without routine use of radiation therapy for patients with locally advanced rectal cancer: a pilot trial. J Clin Oncol. 2014;32:513-8.

17. McCahill LE, Yothers G, Sharif S, et al. Primary mFOLFOX6 plus bevacizumab without resection of the primary tumor for patients presenting with surgically unresectable metastatic colon cancer and an intact asymptomatic colon cancer: definitive analysis of NSABP trial C-10. J Clin Oncol. 2012;30:3223-8.

18. Poultsides GA, Servais EL, Saltz LB, et al. Outcome of primary tumor in patients with synchronous stage IV colorectal cancer receiving combination chemotherapy without surgery as initial treatment. J Clin Oncol. 2009;27:3379-84. 